\title{
Directional Activation of Intestinal Dendritic Cells by an Oral Targeted Multivalent Vaccine
}

\author{
Bikash Sahay, Jennifer L Owen and Mansour Mohamadzadeh*
}

Department of Infectious Diseases and Pathology, Division of Gastroenterology, Hepatology \& Nutrition, Department of Medicine, and Emerging Pathogens Institute, University of Florida, Gainesville, FL 32601, USA

\begin{abstract}
Parenteral injectionis the most common routeof administration for vaccines and therapeutics. Despite their frequent use, needle-based immunizations have several limitations; (i) needle phobias that are common in both adults and children, (ii) the requirement of trained medical personnel to administer vaccines, creating a limitation for mass vaccination, and (iii) accidental needle sticks, a serious concern in both developed and developing countries. Possible alternatives to injections have recently emerged and include (i) dermal and (ii) oral administration of vaccines. Here, we describe a methodology developed in our laboratory using intestinal bacteria that are considered safe for human consumption. Additionally, we have designed a dendritic cell (DC)-targeting sequence that delivers antigens directly to DCs. In this report, we discuss how DC-targeting peptide binding is not limited to human and murine antigen presenting cells; rather, it consistently binds with DCs of different species. Our data suggest that a DC-targeted oral vaccine platform could be used to develop vaccines for a variety of host animals.
\end{abstract}

Keywords: Dendritic cells; Multivalent vaccine

\section{Introduction}

Recently, the search for effective needle-free vaccination has escalated due to heightened concerns of pandemic diseases, bioterrorism, and specific disease eradication programs. Needlefree vaccination could assist withmass vaccination due toits easy and fast delivery, and by providing improved safety, decreasedcost, and reducedvaccination-associated pain, thus, improving compliance. There are two major hurdles in the development of a successful oral vaccine: (i) protection of the antigenof interest from low gastric $\mathrm{pH}$ and digestive enzymes, (ii) and delivery of the antigento dendritic cells (DCs) that are professional antigen presenting cells. To overcome these challenges, two approaches were taken. First, in order to protect the antigen from a harsh gastric environment, LactoBacillus species, including L. acidophilus and L. gasseri, bacteria that can survive and thrivein the gastrointestinal tract, were modified to secrete the antigen of interest in the gut. Secondly, to assist in the deliveryof antigen to professional antigen presenting cells, a twelve amino acid long "targeting" peptide was added tothe immunogenic vaccine subunit $[1,2]$.

\section{Lactobacillus Species as an Antigen Delivery Vehicle}

Mucosal surfaces are the major route of entry for most of the pathogens that cause disease. Thus, vaccines capable of inducing a mucosal immune response can strengthen the defenses at the mucosal layer and protect against infection. Different species of LactoBacillus have been used as dietary componentsofhuman food for centuries and are considered safe for human consumption. Certain species of LactoBacillus are also a part of the normal gut microbiota [3]. Since lactobacillithrivein the low $\mathrm{pH}$ of the stomach, using this bacterium as a vaccine delivery vehicle protects the vaccine subunit from the harsh acidic environment and maintains vaccine bioavailability. Meanwhile, researchers have also developed both inducible and constitutive expression vectors effective in lactobacilli to deliver immunogenic antigens [1,4-6]. Although immunetolerance to a commensal inhabitant of the gastrointestinal mucosa was an initial concern with this strategy, certain lactobacilli such as L. gasseri, are able to overcome tolerance, likely via their strong innate adjuvant properties $[7,8]$. Using
L. gasseri, our laboratory has been able to deliver the protective antigen (PA) of Bacillus anthracis to vaccinated miceto resist this potential agent of bioterrorism [2]. Additionally, another group has used $L$. gasseri for the successful delivery of Salmonella antigens [9].

\section{Targeting Dendritic Cells to Enhance the Immune Reaction}

Dendritic cells (DCs) are the most effective antigen presenting cells in humans and domestic animals, with the unique ability to present antigen and activate naïve $\mathrm{T}$ lymphocytes, thus, playinga criticalrole in the induction of specific primary immune responses. Expression of a varietyof surface receptors, such as C-type lectins (e.g., mannose R, DC-SIGN, DEC-205), Toll-like receptors (TLRs), receptors for the Fc portion of antibodies (FcRs) and complement receptors (CR3, CR4), allow these cells to efficiently bind antigens [10]. Captured antigensaresubsequently processed and efficiently presented to rare antigen specific T lymphocytesdue to the constitutive expression of class II MHC molecules and co-stimulatory/regulatory molecules, including CD40, CD86, and B7-H1 on mature DCs. Therefore, antigen delivered specifically to DCs, as opposed to B lymphocytes or macrophages that require activation to express co-stimulatory molecules,reduces the dose requirement of antigen for immune stimulationand has emerged as a potential vaccination tool to induce protective immune responses [10]. Utilization of traditional receptors (e.g., class II MHC molecules, CD11c, TLRs) for antigen targeting does not resultinefficient delivery of antigen specifically to DCs because many other cell types also express

*Corresponding author: Mansour Mohamadzadeh, Director of the Center for Inflammation \& Mucosal Immunology, Department of Infectious Diseases \& Pathology, Division of Gastroenterology, Hepatology\& Nutrition, Department of Medicine, University of Florida 2015 SW16th Ave, Building 1017, Room: V3-149, Gainesville, FL 32608, USA, E-mail: m.zadeh@ufl.edu

Received June 11, 2013; Accepted June 25, 2013; Published June 28, 2013 Citation: Sahay B, Owen JL Mohamadzadeh M (2013) Directional Activation of Intestinal Dendritic Cells by an Oral Targeted Multivalent Vaccine. J Vaccines Vaccin 4: 192. doi:10.4172/2157-7560.1000192

Copyright: () 2013 Sahay B, et al. This is an open-access article distributed under the terms of the Creative Commons Attribution License, which permits unrestricted use, distribution, and reproduction in any medium, provided the original author and source are credited. 
these receptors and compete with DCs for vaccine binding. With the goal to discover a specific DC-targeting peptide moiety, a phage display library was sequentially absorbed withmonocytes, T cells, B cells, and Langerhans cells and thenscreened for the ability to bindhuman myeloid-derived DCs [11]. The obtained sequence was then tested for its in vitro binding capacity and its application in antigen delivery in a murine model of oral vaccinationby fusing this DC-targeting peptide to PA of Bacillus anthracis $[1,12]$.

Because of their ease of administration, oral vaccine strategies are currently under development or in use for Bordetellabronchiseptica, the primary causative pathogen of the contagious respiratory disease complex, infectious tracheobronchitis or "kennel cough", which is commonly seen in dogs housed together in pet stores, kennels, and animal shelters. This disease can also affect cats and occasionally, immunocompromised humans [13]. Oral therapeutic strategies are also used in rabies control programs that target ownerless pet populations in enzootic areas by vaccinating via palatable bait [14]; for oral vaccination of foals against pneumonia caused by Rhodococcusequi [15]; to protect newly born calves from scours, diarrhea caused by E. coli; and via administration in the drinking water of poultry to prevent or mitigate Newcastle disease, fowl cholera, and avian encephalomyelitis. In fact, poultry are likely the most heavily vaccinated domestic species, with the intensity of production expected to dramatically increase in the next few decades as the economies of countries like China and India improve and poultry consumption increases [16]. To expand upon the applicability of this targeting peptide as an antigen delivery agent in domestic animals, we tested its ability to bind DCs from multiple species of veterinary importance. We found significantly higher binding of DC-peptide to the DCs isolated from peripheral blood from all species tested, including canine, feline, equine, bovine, porcine, and avian species when compared to a non-specific peptide, indicating that this peptide binds to highly conserved regions of its receptor, the identity of which is currently under investigation in our laboratory. These results favorably suggest future use of this particular strategy in the development of veterinarymultivalent vaccines.

\section{Conclusions}

Current vaccination methods employ needle-based administration, which has certain limitations that include the requirement of trained personnel, the risk of accidental needle sticks, and other constraints. To overcome these short comings, we have developed a combinatorial approach that delivers the DC-targeted antigenic protein to intestinal dendritic cells. This approach not only provides a platform for the development of an oral vaccine for human patients, but also allows for the possibility of the generation of oral vaccines against pathogens of veterinary importance.

\section{Acknowledgment}

This work was supported by NIH Grant 1R01AI098833-01.

\section{References}

1. Mohamadzadeh M, Duong T, Sandwick SJ, Hoover T, Klaenhammer TR (2009) Dendritic cell targeting of Bacillus anthracis protective antigen expressed by LactoBacillus acidophilus protects mice from lethal challenge. Proc Natl Acad Sci U S A 106: 4331-4336.

2. Mohamadzadeh M, Durmaz E, Zadeh M, Pakanati KC, Gramarossa M, et al. (2010) Targeted expression of anthrax protective antigen by LactoBacillus gasseri as an anthrax vaccine. Future Microbiol 5: 1289-1296.
3. Walter J (2008) Ecological role of lactobacilli in the gastrointestinal tract: implications for fundamental and biomedical research. Appl Environ Microbiol 74: 4985-4996.

4. Douglas GL, Goh YJ, Klaenhammer TR (2011) Integrative food grade expression system for lactic acid bacteria. Methods Mol Biol 765: 373-387.

5. Duong T, Miller MJ, Barrangou R, Azcarate-Peril MA, Klaenhammer TR (2011) Construction of vectors for inducible and constitutive gene expression in LactoBacillus. Microb Biotechnol 4: 357-367.

6. Kajikawa A, Nordone SK, Zhang L, Stoeker LL, LaVoy AS, et al. (2011) Dissimilar properties of two recombinant LactoBacillus acidophilus strains displaying Salmonella FliC with different anchoring motifs. Appl Environ Microbiol 77: 6587-6596.

7. Mohamadzadeh M, Klaenhammer TR (2008) Specific LactoBacillus species differentially activate Toll-like receptors and downstream signals in dendritic cells. Expert Rev Vaccines 7: 1155-1164.

8. Mohamadzadeh M, Olson S, Kalina WV, Ruthel G, Demmin GL, et al. (2005) Lactobacilli activate human dendritic cells that skew $T$ cells toward $T$ helper 1 polarization. Proc Natl Acad Sci U S A 102: 2880-2885.

9. Stoeker L, Nordone S, Gunderson S, Zhang L, Kajikawa A, et al. (2011) Assessment of LactoBacillus gasseri as a candidate oral vaccine vector. Clin Vaccine Immunol 18: 1834-1844.

10. Banchereau J, Briere F, Caux C, Davoust J, Lebecque S, et al. (2000) Immunobiology of dendritic cells. Annu Rev Immunol 18: 767-811.

11. Curiel TJ, Morris C, Brumlik M, Landry SJ, Finstad K, et al. (2004) Peptides identified through phage display direct immunogenic antigen to dendritic cells. J Immunol 172: 7425-7431.

12. Erskine CL, Krco CJ, Hedin KE, Borson ND, Kalli KR, et al. (2011) MHC class II epitope nesting modulates dendritic cell function and improves generation of antigen-specific CD4 helper T cells. J Immunol 187: 316-324

13. Register KB, Sukumar N, Palavecino EL, Rubin BK, Deora R (2012) Bordetella bronchiseptica in a paediatric cystic fibrosis patient: possible transmission from a household cat. Zoonoses Public Health 59: 246-250.

14. Hicks DJ, Fooks AR, Johnson N (2012) Developments in rabies vaccines. Clin Exp Immunol 169: 199-204.

15. Whitehead AE, Parreira VR, Hewson J, Watson JL, Prescott JF (2012) Development of a live, attenuated, potential vaccine strain of $R$. equ expressing vapA and the virR operon, and virulence assessment in the mouse. Vet Immunol Immunopathol 145: 479-484.

16. Wu Z, Kaiser $P$ (2011) Antigen presenting cells in a non-mammalian mode system, the chicken. Immunobiology 216: 1177-1183. 